MINI-SYMPOSIUM

\title{
Aortic intramural haematoma: remarks and conclusions
}

\author{
A Evangelista
}

Heart 2004;90:379-380. doi: 10.1136/hrt.2003.027649

A ortic intramural haematoma (IMH) originates from spontaneous rupture of the aorta vasavasorum or from a penetrating atherosclerotic ulcer. Recent advances in imaging techniques have significantly aided its diagnosis, placing the incidence between $10-30 \%$ of acute aortic syndromes. Clinical presentation may be identical to that of classical dissection. IMH affects patients with more atherosclerotic risk factors and older age than aortic dissection, and it is located in the descending aorta in $60-70 \%$ of cases.

\section{IMAGING TECHNIQUE INFORMATION}

Although the diagnostic sensitivity seems to be similar among computed tomography (CT), magnetic resonance imaging (MRI), and transoesophageal echocardiography (TOE), the diagnosis of IMH is more complex than that of classical dissection, with at least two diagnostic techniques being performed in most cases. In the International Registry of Aortic Dissection, the CT-TOE combination was the most used. Nevertheless, MRI has the advantage of permitting detection of new bleeds in the aorta wall, which may be important in subacute and chronic phases.

Imaging techniques provide significant prognostic information. Concurring with data reported by others, ${ }^{1-3}$ maximum aorta diameter in our series of 64 patients was an important predictor of adverse clinical events including death. ${ }^{4}$ Fluid extravasation, pericardial and pleural effusion, and mediastinal haemorrhage, more frequent in IMH than in classical dissection, worsen the prognosis. However, progressive accumulation of a large amount of pleural effusion is not in itself an indication for surgical treatment.

\section{NATURAL HISTORY AND PREDICTIVE FACTORS OF COMPLICATIONS}

Short term mortality depends essentially on IMH location, and is higher when the ascending, rather than descending, aorta is involved. In our series, overall IMH mortality in the first three months was $19 \%$, similar to that of other series, with proximal involvement and maximum aortic diameter more than $50 \mathrm{~mm}$ being variables of independent prognostic value. ${ }^{4}$ Recent work by Dr Song ${ }^{3}$ found that haematoma thickness more than $11 \mathrm{~mm}$ in the ascending aorta predicted adverse outcomes with $89 \%$ sensitivity.

There is a paucity of information on the long term evolution of IMH, although several variables such as younger age, absence of long term oral $\beta$ blocker treatment, larger aortic diameter, and the presence of penetrating aortic ulcers have been related to worse prognosis. In our experience, TOE is very useful for differentiating penetrating aortic ulcer from ulcer-like images. These images, secondaries to focal dissections with small entry tears, are frequently detected with contrast CT in the subacute phase of IMH and have better prognosis than penetrating atherosclerotic ulcers.

In our series ${ }^{5}$ of 50 intramural haematomas followed for a mean of four years, $34 \%$ reabsorbed without complications, $12 \%$ progressed to classical dissection, $24 \%$ to pseudoaneurysm, and $30 \%$ evolved to fusiform or saccular aneurysm of the aorta. A normal aortic diameter in the acute phase was

the best predictor of IMH regression without complications, and absence of echolucent areas and atherosclerotic ulcerated plaque were associated with evolution to aortic aneurysm. IMH thickness was significantly smaller in the reabsortion group.

\section{MANAGEMENT}

It is currently believed that the therapeutic strategy for IMH should be the same as that for aortic dissection. In a metaanalysis $^{6}$ of series published up to 1999, mortality of type A IMH treated medically was very high (56\%). However, Asian series showed low death rates in proximal IMH treated medically. ${ }^{18}$ In agreement with Dr Nienaber's comments, at least half of the patients with type A initially treated medically require pericardiocentesis or surgery in Asian series. However, the evolution of type A IMH appears to be more benign than that of classical dissection. In a recent study, Kaji and colleagues ${ }^{8}$ showed that medical treatment with timed surgical repair in cases with progression can also be a rational therapeutic strategy in patients with type A IMH. Careful follow up imaging study during acute and subacute phases is absolutely necessary. Transthoracic echocardiography was performed daily and TOE within three days after the diagnosis and once a week until the third week. CT was repeated once a week. In this series, $43 \%$ of type A IMH required surgery. With this strategy, it is essential that diagnostic imaging modalities and urgent surgical intervention are readily available. The subgroup of patients at low risk for complications should be defined before treatment, other than surgery, is indicated, particularly in younger patients with low comorbidity.

Type B IMH should be treated with $\beta$ blockers, closely followed up with imaging techniques, until complication-free absorption of the IMH is observed. The subgroup with progressive aortic dilatation or with images of localised (ulcer-like) dissection should be closely followed and treated more aggressively. Endovascular placement of stent grafts to cover some extent of IMH appears to be a promising treatment for this type of complication; however, it remains to be confirmed by results of large clinical series.

Correspondence to: A Evangelista, MD, FESC, Servei de Cardiologia, Hospital Vall d'Hebron, PVall D'Hebron 1 19, 08035 Barcelona, Spain; evangel@hg.vhebron.es

\section{REFERENCES}

1 Kaji S, Nishigami K, Akasaka T, et al. Prediction of progression or regression of type A aortic intramural hematoma by computed tomography. Circulation 1999; 100(suppl II):281-6.

2 Sueyoshi E, Imada T, Sakamoto I, et al. Analysis of predictive factors for progression of type B aortic intramural hematoma by computed tomography. J Vasc Surg 2002;35:1179-83.

Abbreviations: $\mathrm{CT}$, computed tomography; IMH, intramural haematoma; MRI, magnetic resonance imaging; TOE, transoesophageal echocardiography 
3 Song JM, Kim HS, Dong JK, et al. Usefulness of the initial non-invasive imaging study to predict the adverse outcomes in the medical treatment of acute type A aortic intramural hematoma. Circulation 2003;108(suppl II):324-8.

4 Evangelista A, Domínguez R, Sebastià C, et al. Prognostic value of clinical and morphologic findings in short-term evolution of aortic intramural haematoma. Therapeutic implications. Eur Heart J 2004;25:81-7.

5 Evangelista A, Domínguez R, Sebastià C, et al. Long-term follow-up of aortic intramural hematoma. Predictors of outcome. Circulation 2003;108:583-9.
6 Marai $R$, Rerkpattanapipat $P$, Jacobs LE, et al. Meta-analysis of 143 reported cases of aortic intramural hematoma. Am J Cardiol 2000;86:664-8.

7 Song JK, Kim HS, Kang DH, et al. Different clinical features of aortic intramural hematoma versus dissection involving the ascending aorta. J Am Coll Cardiol 2001:37:1604-10.

8 Kaji S, Alasaka T, Horibata Y, et al. Long-term prognosis of patients with type $A$ aortic intramural hematoma. Circulation 2002; 106(suppl I):248-52.

\section{IMAGES IN CARDIOLOGY}

\section{Hypertrophic cardiomyopathy with persistent ST segment elevation simulating acute myocardial} infarction

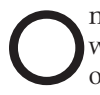

May 2000, a 67 year old woman was admitted to the hospital because of right upper eyelid ptosis. She was normotensive and had never experienced chest pain, dyspnoea or other symptoms suggestive of heart disease. A routine 12 lead ECG (upper panel, A) revealed an ST segment elevation with associated negative T waves in several leads, suggesting a possible acute myocardial infarction. Physical examination did not reveal any abnormal heart finding, and cardiac enzymes were negative. Echocardiography (lower panels $\mathrm{A}$ and $\mathrm{B}$ : LA, left atrium; LV, left ventricle; RA, right atrium; RV, right ventricle) revealed a pronounced and generalised left ventricular hypertrophy with normal wall motion and absence of gradient across the left ventricular outflow tract. Several ECGs obtained in the following days were unchanged. The coronary angiogram showed normal coronary arteries. Accordingly, the apparent acute myocardial infarction pattern was likely to be dependent on hypertrophic cardiomyopathy. The patient was followed up and no changes in the ECG pattern were observed in the ensuing three years (upper panel, B).

This case shows that hypertrophic cardiomyopathy can be associated with an ECG falsely suggesting an acute myocardial infarction.

F Luzza, S Careri, G Oreto francescoluzza@tiscali.it
A
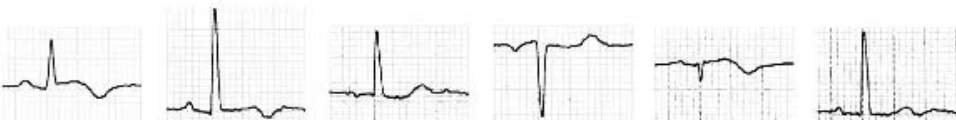

$18 \mathrm{May}$

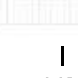

2000

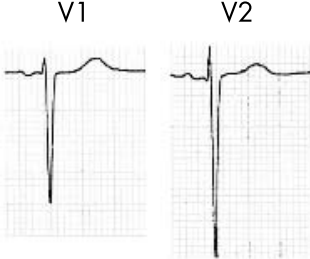

V3

aVR

$\mathrm{aVL}$

aVF

V5

V6
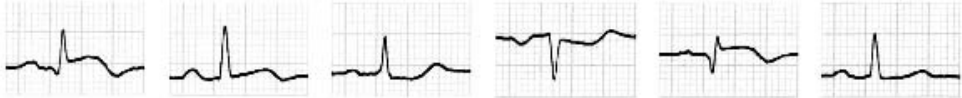

11 June

I 1

$\stackrel{\text { II }}{\text { V2 }}$

III
V3

aVR

$\mathrm{aVL}$
$\mathrm{V} 5$

aVF
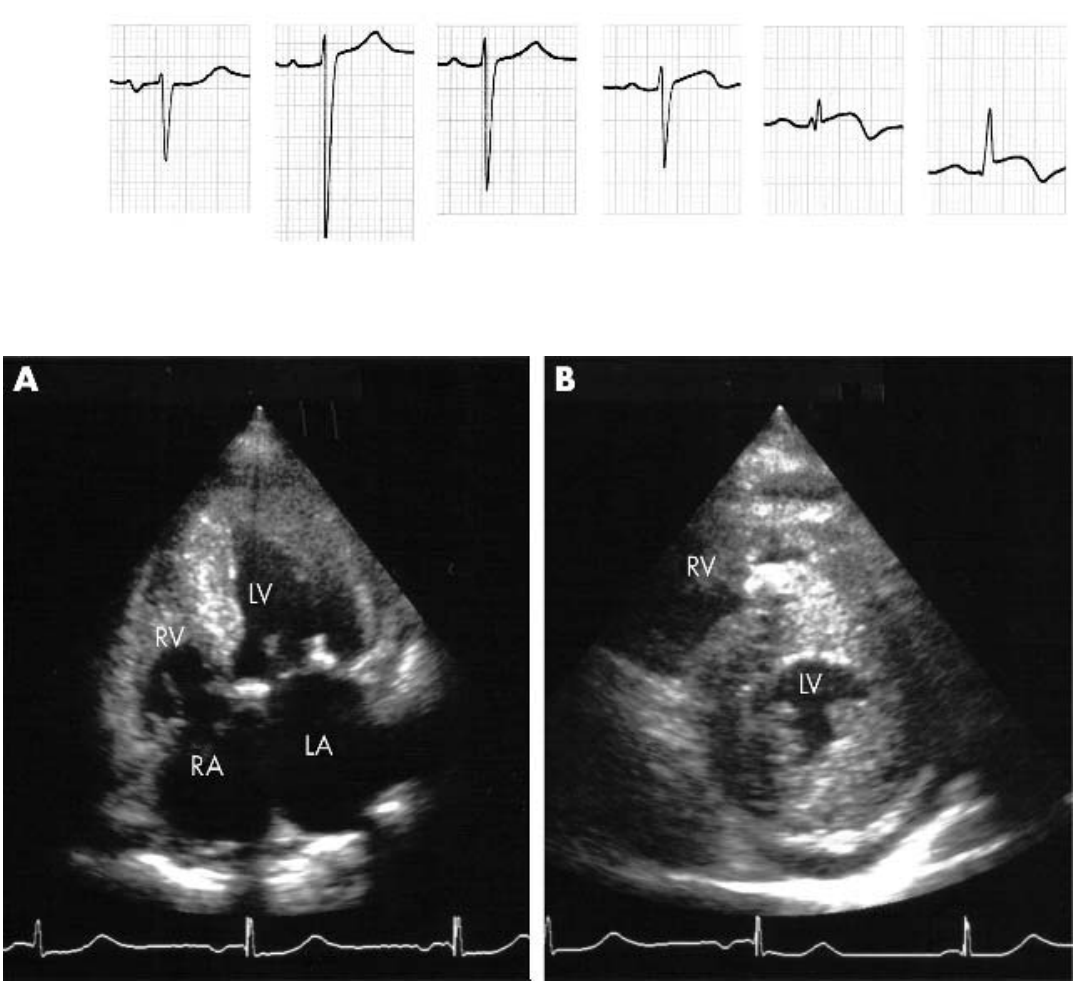

B

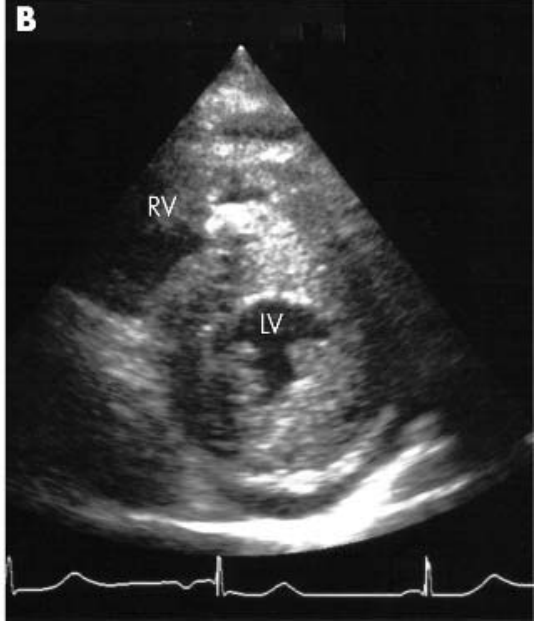

